\title{
ON THE MEANS OF QUASIREGULAR AND QUASICONFORMAL MAPPINGS
}

\author{
DAVID JERISON AND ALLEN WEITSMAN ${ }^{1}$
}

\begin{abstract}
Two theorems are given regarding the means of quasiconformal and quasiregular mappings. Together they show that the principle of subordination for means of analytic functions has no analog, at least in the case of plane quasiregular mappings.
\end{abstract}

I. Introduction. Let $B^{n}(r)=\left\{x \in \mathbf{R}^{n}:|x|<r\right\}, B^{n}=B^{n}(1)$, and $f(x)$ be a quasiregular mapping of $B^{n}$. Then $f$ is continuous, $f \in W_{n, \text { loc }}^{1}\left(B^{n}\right)$, and for some constant $K(1 \leqslant K<\infty)$,

$$
\left\|f^{\prime}(x)\right\|^{n} \leqslant K J_{f}(x) \quad \text { a.e.; }
$$

here $J_{f}$ is the Jacobian determinant, $f^{\prime}$ the Jacobian matrix, and

$$
\left\|f^{\prime}(x)\right\|=\sup _{|e|=1}\left|f^{\prime}(x) \cdot e\right| .
$$

If in addition $f$ is injective, $f$ is called quasiconformal.

The theory of quasiregular mappings is often referred to as a natural extension of analytic function theory, and indeed many basic properties of analytic functions extend in some form. A brief account of this along with relevant definitions can be found in the survey [5].

In the present note we shall present two results which together show that the subordination principle for means, which plays a prominent role in the theory of analytic functions [2, p. 10], has no counterpart in the theory of quasiregular mappings.

We shall use polar coordinates, denoting by $d \sigma$ the surface element on $\partial B^{n}$, and $d V$ the volume element on $\mathbf{R}^{n}$.

THEOREM 1. Let $f$ be a quasiconformal mapping of $B^{n}$. Then there exists a $p=p(n, K)$ such that

$$
\limsup _{R \rightarrow 1} \int_{\partial B^{n}}|f(R \zeta)|^{p} d \sigma(\zeta)<\infty .
$$

In the case $n=2$ and $f$ conformal, any $p<\frac{1}{2}$ suffices in (1.3) [2, p. 50]; our proof does not give this. Also if $n=2$ and $f$ is conformal, then the classical subordination principle says that the $p$ th mean is bounded for any analytic $g$ in $B^{2}$, whose range is

Received by the editors December 7, 1980.

1980 Mathematics Subject Classification. Primary 30C60, 30C75, 30 C80.

Key words and phrases. Quasiconformal, quasiregular, means.

${ }^{1}$ Research of the authors supported by the National Science Foundation. 
contained in $f\left(B^{2}\right)$. Our next example shows, at least in the case $n=2$, that there is no corresponding effect for quasiregular mappings.

THEOREM 2. There exists a quasiregular function $g$ mapping $B^{2}$ to a half plane such that if $\Phi(t): \mathbf{R} \rightarrow \mathbf{R}$ is any increasing function with $\Phi(t) \rightarrow \infty$ as $t \rightarrow \infty$ then

$$
\lim _{r \rightarrow 1} \int_{0}^{2 \pi} \Phi\left(\left|g\left(r e^{i \theta}\right)\right|\right) d \theta=\infty
$$

It would be nice to have an example for arbitrary $n$.

II. Proof of Theorem 1. Let $f=\left(f_{1}, \ldots, f_{n}\right)$. We may assume without loss of generality that $f(0)=0$ and thus $|f|>\varepsilon$ for some $\varepsilon>0$, if $|x|>\frac{1}{2}$. In the course of this proof we shall use the letter $C$ to denote constants which may not be the same at each occurrence, but which do not depend upon $R$ and $p$.

Since $f$ is absolutely continuous on almost every ray from the origin $[4$, p. 108] we have for any $p>0$ and $\frac{1}{2}<R<1$

$$
\begin{aligned}
\int_{\partial B^{n}}|f(R \zeta)|^{p} d \sigma(\zeta)-\int_{\partial B^{n}}\left|f\left(\frac{1}{2} \zeta\right)\right|^{p} d \sigma(\zeta) \\
\quad=\int_{\partial B^{n}} \int_{1 / 2}^{R} \frac{\partial|f|^{p}}{\partial r} d r d \sigma \\
=p \int_{1 / 2}^{R} \int_{\partial B^{n}}|f|^{p-2}\left(f_{1} \frac{\partial f_{1}}{\partial r}+\cdots+f_{n} \frac{\partial f_{n}}{\partial r}\right) d \sigma d r \\
\quad \leq p \int_{1 / 2}^{R} \int_{\partial B^{n}}|f|^{p-1}\left(\left(\frac{\partial f_{1}}{\partial r}\right)^{2}+\cdots+\left(\frac{\partial f_{n}}{\partial r}\right)^{2}\right)^{1 / 2} d \sigma d r .
\end{aligned}
$$

Applying (1.1), (1.2) and then Hölder's inequality we obtain for $\frac{1}{2}<R<1$

$$
\begin{aligned}
\int_{\partial B^{n}}|f(R \zeta)|^{p} d \sigma(\zeta)< & K^{1 / n} p \int_{1 / 2}^{R} \int_{\partial B^{n}}|f|^{p-1} J_{f}^{1 / n} d \sigma d r+C \\
< & K^{1 / n} p\left(\int_{1 / 2}^{R} \int_{\partial B^{n}}|f|^{-n(p+1)} J_{f} r^{n-1} d \sigma d r\right)^{1 / n} \\
& \cdot\left(\int_{1 / 2}^{R} \int_{\partial B^{n}}|f|^{2 p n /(n-1)} r^{-1} d \sigma d r\right)^{(n-1) / n}+C .
\end{aligned}
$$

Now,

$$
\begin{aligned}
\int_{1 / 2}^{R} \int_{\partial B^{n}}|f|^{-n(p+1)} J_{f} r^{n-1} d \sigma d r & =\int_{B^{n}(R)-B^{n}(1 / 2)}|f|^{-n(p+1)} J_{f} d V \\
& <\int_{R^{n}-B^{n}(\varepsilon)}|x|^{-n(p+1)} d V(x)<\infty .
\end{aligned}
$$

Now, $f\left(B^{n}\right) \neq R^{n}[4$, p. 53] so if $\tau$ is an omitted value we have [3, p. 73] $|f(x)|<|\tau|+C|\tau|((1+r) /(1-r))^{\alpha}, \alpha=\alpha(K, n)$ and thus

$$
\int_{1 / 2}^{R} \int_{\partial B^{n}}|f|^{2 p n /(n-1)} r^{-1} d \sigma d r<C \int_{1 / 2}^{R}(1-r)^{-2 p n \alpha /(n-1)} d r+C .
$$

Since the right-hand side of $(2.3)$ is finite for sufficiently small $p>0$, the result follows from (2.1)-(2.3). 
III. Proof of Theorem 2. For a Borel subset $E$ of $[0,2 \pi)$, let $\chi_{E}$ be its characteristic function, and $|E|=\int \chi_{E} d \theta$.

From [1, p. 139] we obtain a quasiconformal mapping $\phi$ of $B^{2}$ onto itself such that on $\partial B^{2}$ the measure $\mu$ defined by $\mu(E)=|\phi(E)|$ is singular with respect to $d \theta$. Let $E \subseteq \partial B^{2}$ such that $|E|=0, \mu(E)>0$, and let $V_{1} \supseteq V_{2} \supseteq \ldots$ be open subsets of $[0, \pi)$ such that $\left|V_{n}\right|=2^{-n}$ and $\bigcap_{n} V_{n} \supseteq E$. Define $f(\theta)=\Sigma \chi_{V_{n}}(\theta)$. Then, $f \in L^{2}(d \theta)$ since

$$
\begin{aligned}
\int_{0}^{2 \pi}|f|^{2} d \theta & \leqslant \sum_{n=1}^{\infty} \int_{V_{n}-\cup_{j>n} V_{j}}\left(\sum_{k=1}^{\infty} \chi_{V_{k}}\right)^{2} d \theta \\
& <\sum_{n=1}^{\infty} \int_{V_{n}} n\left(\sum_{k=1}^{n} \chi_{V_{k}}\right) d \theta<\sum_{n=1}^{\infty} n^{2} 2^{-n}<\infty .
\end{aligned}
$$

Thus, we may take the Poisson integral of $f$ in $B^{2}$, which we continue to denote by $f$. Let $\tilde{f}$ be a conjugate of $f$, and $F=f+i \tilde{f}$.

If $g=F \circ \phi^{-1}$ then, $g$ is quasiregular, and $\Re$ e $g>0$ in $B^{2}$, and

$$
\begin{aligned}
\int_{0}^{2 \pi} \Phi\left(\left|g\left(r e^{i \theta}\right)\right|\right) d \theta & \geqslant \int_{0}^{2 \pi} \Phi\left(f \circ \phi^{-1}\left(r e^{i \theta}\right)\right) d \theta \\
& \geqslant \int_{\phi\left(V_{n}\right)} \Phi\left(f \circ \phi^{-1}\left(r e^{i \theta}\right)\right) d \theta \quad \forall n=1,2, \ldots
\end{aligned}
$$

Now, the right-hand side of (3.1) is bounded below by $\int_{\phi\left(V_{n}\right)} \Phi\left(h_{n} \circ \phi^{-1}\left(r e^{i \theta}\right)\right) d \theta$ where $h_{n}$ is the Poisson integral of $n \chi_{V_{n}}$. But $h_{n}\left(r e^{i \theta}\right)$ is continuous for $0<r<1$, $\theta \in V_{n}$ since $V_{n}$ is open, and $\phi^{-1}$ is continuous on $\bar{B}^{2}$. Thus, the limit of $\int_{\phi\left(V_{n}\right)} \Phi\left(h_{n} \circ \phi^{-1}\left(r e^{i \theta}\right)\right) d \theta$ exists as $r \rightarrow 1$. Since

$$
\int_{\phi\left(V_{n}\right)} \Phi(n) d \theta=\Phi(n) \mu\left(V_{n}\right) \geqslant \Phi(n) \mu(E), \quad 0<\mu(E),
$$

and $\Phi(n)$ can be made arbitrarily large, it follows that

$$
\int_{0}^{2 \pi} \Phi\left(\left|g\left(r e^{i \theta}\right)\right|\right) d \theta \rightarrow \infty \quad \text { as } r \rightarrow 1
$$

\section{REFERENCES}

1. A. Beurling and L. Ahlfors, The boundary correspondence under quasiconformal mappings, Acta Math. 96 (1956), 125-142.

2. P. Duren, Theory of $H^{p}$ spaces, Academic Press, New York, 1970.

3. R. Miniowitz, Distortion theorems for quasiregular mappings, Ann. Acad. Sci. Fenn. 4 (1978/79), 63-74.

4. J. Väisälä, Lectures on n-dimensional quasiconformal mappings, Lecture Notes in Math., vol. 229, Springer-Verlag, Berlin and New York, 1971.

5. 685-691. A survey of quasiregular maps in $\mathbf{R}^{n}$, Proc. Internat. Congr. Math., Helsinki (1978), pp.

Department of Mathematics, University of Chicago, Chicago, Illinots 60637

Department of Mathematics, Purdue University, West Lafayette, Indiana 47907 (Current address of Allen Weitsman)

Current address (David Jerison): Department of Mathematics, Massachusetts Institute of Technology, Cambridge, Massachusetts 02139 